\title{
Erratum: Complete one-loop matching for a singlet scalar in the Standard Model EFT
}

Minyuan Jiang, ${ }^{a, b}$ Nathaniel Craig, ${ }^{b, c}$ Ying-Ying $\mathbf{L i}^{d, c}$ and Dave Sutherland ${ }^{b}$

${ }^{a}$ Department of Physics, Nanjing University, Nanjing 210098, P.R.C.

${ }^{b}$ Department of Physics, University of California, Santa Barbara, CA 93106, U.S.A.

${ }^{c}$ Kavli Institute for Theoretical Physics, Santa Barbara, CA 93106, U.S.A.

${ }^{d}$ Department of Physics, The Hong Kong University of Science and Technology, Clear Water Bay, Kowloon, Hong Kong S.A.R., P.R.C.

E-mail: dg1522023@smail.nju.edu.cn, ncraig@physics.ucsb.edu, ylict@connect.ust.hk, dwsuth@ucsb.edu

ERRATUM TO: JHEP02(2019)031

ArXiv EPrint: 1811.08878 
There are terms missing from the one-loop matching calculation of [1].

One, the expressions for $c_{H}$ and $c_{H \square}$ should be modified, such that (3.1), (3.2), and (A.19) respectively in [1] read

$$
\begin{aligned}
16 \pi^{2} \delta c_{H}= & -\frac{\kappa^{3}}{12}+\frac{-48 A^{2} \kappa \lambda+36 A^{2} \lambda^{2}+22 A^{2} \kappa^{2}-2 A^{2} \kappa \lambda_{\phi}-A \kappa^{2} \mu}{4 M^{2}} \\
& +\frac{39 A^{4} \kappa-3 A^{4} \lambda_{\phi}+36 A^{3} \mu \lambda-30 A^{3} \kappa \mu+2 A^{3} \mu \lambda_{\phi}-36 A^{4} \lambda+3 A^{2} \mu^{2} \kappa}{6 M^{4}} \\
& +\frac{-8 A^{6}-18 A^{5} \mu+12 A^{4} \mu^{2}-2 A^{3} \mu^{3}}{12 M^{6}}+\left[\frac{36 A^{2} \kappa \lambda-36 A^{2} \lambda^{2}-12 A^{2} \kappa^{2}+A^{2} \kappa \lambda_{\phi}}{4 M^{2}}\right. \\
& \left.+\frac{-18 A^{4} \kappa-36 A^{3} \mu \lambda+12 A^{3} \kappa \mu-A^{3} \mu \lambda_{\phi}}{12 M^{4}}+\frac{2 A^{5} \mu}{4 M^{6}}\right] \log \frac{M^{2}}{\mu^{2}}, \\
16 \pi^{2} \delta c_{H \square}= & -\frac{\kappa^{2}}{24}+\frac{-27 A^{2} \lambda+34 A^{2} \kappa-6 A^{2} \lambda_{\phi}-5 A \kappa \mu}{12 M^{2}}+\frac{26 A^{4}-32 A^{3} \mu+11 A^{2} \mu^{2}}{24 M^{4}} \\
& +\left[\frac{A^{2} \lambda-4 A^{2} \kappa+A^{2} \lambda_{\phi}}{2 M^{2}}+\frac{-2 A^{4}}{2 M^{4}}\right] \log \frac{M^{2}}{\mu^{2}}, \\
16 \pi^{2} c_{H \square}= & \frac{1}{72}\left(162 \lambda-93 g^{2}-31 g^{\prime 2}-54 \kappa+\frac{18 A \mu}{M^{2}}+\left(90 g^{2}+30 g^{\prime 2}-36 \lambda\right) \log \frac{M^{2}}{\mu^{2}}\right) \frac{A^{2}}{M^{2}} \\
& +16 \pi^{2}\left(\delta c_{H \square}+\left.\delta c_{H \square}\right|_{\text {shift }}\right) .
\end{aligned}
$$

Cf. expressions (3.9) and (3.11) in [2] for the same calculation.

Two, an additional Warsaw basis operator

$$
\mathcal{O}_{H u d}=\left(\tilde{H}^{\dagger} i D_{\mu} H\right)\left(\bar{u} \gamma^{\mu} d\right)
$$

is generated at one-loop, and should be included in table 1 of [1]. Its Wilson coefficient

$$
16 \pi^{2} c_{H u d}=\left(-\frac{5}{4}+\frac{1}{2} \log \frac{M^{2}}{\mu^{2}}\right) \frac{A^{2}}{M^{2}} y_{u}^{\dagger} y_{d},
$$

should be added to the list in appendix A of [1].

\section{Acknowledgments}

We are grateful to the authors of [2] for pointing out the corrections to $c_{H}$ and $c_{H \square}$, and to B. Summ for the corrections to $c_{H u d}$.

Open Access. This article is distributed under the terms of the Creative Commons Attribution License (CC-BY 4.0), which permits any use, distribution and reproduction in any medium, provided the original author(s) and source are credited. 


\section{References}

[1] M. Jiang, N. Craig, Y.-Y. Li and D. Sutherland, Complete One-Loop Matching for a Singlet Scalar in the Standard Model EFT, JHEP 02 (2019) 031 [arXiv:1811.08878] [INSPIRE].

[2] U. Haisch, M. Ruhdorfer, E. Salvioni, E. Venturini and A. Weiler, Singlet night in Feynman-ville: one-loop matching of a real scalar, JHEP 04 (2020) 164 [Erratum JHEP 07 (2020) 066] [arXiv: 2003. 05936] [INSPIRE]. 